\title{
BAND XXXXVII.
}

I. Beitrag zu den elektro-chemischen Merkwürdigkeiten der salpetersauren Silberlösung;

con G. Th. Fechner ${ }^{2}$ ).

Die folgende Abbandlung hat den Zweck, theils einige an sich interessante Thatsachen mitzutheilen, theils an einigen Beispielen den bisher nicbt berücksichtigten $\mathrm{Za}$ sammenhang zwischen verschicdenen Umständen zu zeigen, die bei elektro-chemischen Versuchen Anwendung erleiden.

Mebrere der hieher gehörigen Versuche werden al- . lerdings eine andere Deutung, als ich ihncn gegeben, vou Denen erfahren, welche das auf Rechnung von Gegenspannungen oder von Elektricitäts-Erregung durch Berührung von Flussigkeiten unter einander oder unit festen Körpern zu schreiben geneigt sind, was ich auf Rechnung materieller Veränderungen der Metalle durch die Flüssigkeiten, wenn oicht allein, doch grofsentheils schreibe. Da die vorliegende Abhandlung vicbt bestimmt ist, über das Für oder Wider dieser Ansichien zu entscheiden, so überlasse ich Jedem gern, den Ausdruck der Thatsachen, den ich nach meiner Ansicht gestellt habe, in die Sprache seiner eigenen Ansicht zu übersetzen. Allerdings dürfte man Mehreres darunter vorfinden, was :ich jenen Ansichten nur gezwungen fügen wird.

Als Multiplicatoren dienten bei den folgenden Ver-

1) In meiner letzten Abhandlung über dic Vortheile langer Multiplicatoren (dicse Ann., Bd. XXXXV S. 232 fl:) bitte ich, gelegentlich Folgendes zu berichtigen: S. $236 \mathrm{Z} .16$ v. u. st. Wirkungen l. Windungen. - S. 243 Z. 15 v. o. st. au l. von. - S. 245 Z. 12 v. u. streiche das Wort nicht.

Poggendorf?: Annal. Bd. XXXXVII. 
suchen stets die beiden längeren Exemplare, die ich in weiner letzten Abhandlung (diese Annalen, Bd. XXXXV S. 232) beschrieben habe, und zu allen messenden Versuchen namentlich der längste, mit $L$ bezeichnete, ausgenommen zu den, unter No. 3 beschriebenen, zu welchen der Multiplicator $K$ diente. Diese Ansrendung langer Multiplicatoren hat unstreitig nicht nur die Deutlichkeit der Wirkungen befördert, sondern es anch möglich gemacht, bei einer Schicht Flüssigkeit, die nie über einige Linien Höhe (bei einem Abstande der Platten von ungefähr 1 Zoll) betrug, Resultate zu erbalten, die mindestens sehr wenig von Veränderungen des UebergangsWiderstandes afficirt seyn können.

Wo ich bei Maafsen, die mittelst der OscillationsMethode (bei senkrechter Lage der Doppeluadel gegen die Windungen) genommen siud, nicht gleich die berechneten Kräfte selbst mittheile, kann man dieselben mit Hülfe des Datums berechnen, dafs die Doppelradel unter dem blofsen Einflufs der Erde 92 Zeittheile (Drittel-Secunden) zu je 2 Oscillationen, dereu Zeit in Folgenden immer angegeben ist, brauchte.

In Betreff der im Folgenden vorkommenden Versuche mit ungleichzeitigem Eintauchen hounogener Metalle bennerke ich, dafs ohne Ausnahme nach geschehener Anstellung eines Versuchs derselbe mit neugereinigten und verwechselten Platten wiederholt wurde, un jeden Einflufs eines ursprünglichen Mangels au Homogenität auszuschliefsen.

Unter starker Silberlösung verstehe ich im Folgenden in allen Fällen, wo nichts Anderes bemerkt ist (und diefs ist nur unter No. 2 der Fall), eine solche, welche 1 Gewichtstheil des Salzes auf 12 Gewichistheile Wasser enthält. Diese Lösung ist auch den Verdünnungen als Einheit zu Grunde gelegt. Sie reagirte etwas säuerlich.

1) Eine bemerkenswerthe Mannichfaltigkeit von Formin, unter welchen sich das Silber niederschlagen kann, 
bietet die Făllung desselben durch Zinn dar. Man giefse in ein Uhrglas etwas starke Silberlösung und lege ein Stückchen blankes Zinn hinein, am besten den cylinderförmigen Abschnitt eines Zinudralıts auf eine seiner Basen. So wie man es hineinlegt, schwärzt es sich, umgiebt sich dann sofort mit einem grauen Bart, sehr bald aber - und diefs ist der frappanteste Theil des Phänomens - fangen von dem grauen Barte an, an der Oberfläche der Flüssigkeit, zarte weilse Theilchen radieuartig mit einer schr bemerkenswerthen Abstuisungsbewegung in die Flüssigkeit hineinzustrahlen, so dafs das Ganze das Ansehen einer kleinen weirsen Sonne erbält. Dem Anschein nach stellt sich die weifse Substanz als Zinnoxyd dar; da sic sich indefs an Lichte dunkler färbt, so mülste sie in diesem Fall wenigstens eine Silberverbindung beigemengt enthalten. Nicht minder merkwürdig als die Erzeugung dieser Substanz ist der Umstand, dals sie stets blofs eine gewisse Periode iundurch sich bildet. Allmälig hört die Bildung ganz auf, dic äufseren Theilchen werden durch den Einflufs des Lichts dunkelviolet und unscheinbar, und es bleibt blofs noch eine Zeit lang ein weifser Ring un den grauen Bart sichtbar, der sich von jetzt an in metallglänzenden Nadeln oder Blăttern fortsetzt, die unter dem weilsen Ringe bervorwachsen und allmälig die ganze Flüssigkeit auskrystallisiren. In einem Falle sah ich statt dieser glänzendeu Nadeln ganz schwarze Dendriten das Uhrglas überziehen, habe aber in einein Gefälse dieser Art dieselbe Erscheinung nicht wieder hervorzubringen vermocht. Dagegen erhält man eiden schwarzen Niederschlag, jedoch mehr in Gestalt einer amorphen Masse, mit Sicherheit, wenn man in ein Glasgefäls mit flachem, aber in der Mitte sich ein wenig aufwrärts wölbendem Boden sehr wenig Silberlösung giefst, so dafs sie die mittlere Wölbung haum oder gar nicht überdeckt, und eine Zinnplatte am Rande des Gefälses hineinstellt. Nachdew der graue Bart und die weifsen Strah- 
len wic vorbin entstanden sind, quillt unter der weifsen Materie die schwarze bervor, offers einen Bart von grauen Dendriten vor sich herschiebend. Mauchmal ist die schwarze Farbe dieses Niederschlags ausnehmend tief und dann durch den Gegensalz zur weifsen Materie sehr bemerkenswerth. Besonders glaube ich diefs dann gefunden zu baben, wenn unter den angegebenen Unständen das Zinn mit Zink (in einem Abstand ron 1 bis $1 \div$ Zoll davon stebend) zur Kette geschlossen war; doch kann ich nicht rersichern, dafs diefs gerade wesentlich ist. Auch bein Zink quillt nach einiger Zeit in der sehr seicht stelsenden Auflüsung unter dem grauen Barte eine schwarze Materie bervor, welche schnell vorschreitet; aber nie zeigt sich dieselbe weifse Materie, als am Zinn, wo sie bei allen Formen des Versuchs constant ist.

2) Die Schliefsung des Zinks mit Zinn in starker Silberlösung (sowobl bei $1 \mathrm{Th}$. Salz in $8 \mathrm{Th}$., als in 12 Th. Wasser) ist noch von besonderen inerkwürdigen Umständen beğleitet. Bei oft wiederholten Versuchen habe ich nämlich Folgendes constant wahrgenommen. (Das Zink war destillirtes, das Zinn gutes englisches. Beide Platten standen in 1 bis $1 \frac{1}{2}$ Zoll Abstand. Die Flüssigkeitsschicht hatte, wie inmer, nur einige Lin. Hölse.) Wenn die Kelle durch das Einlauchen der an den Enden des Multiplicators befestigten Platten geschlossen wird "), so wacht in Moment des Eintauchens die Nadel des Multiplicators einen einzigen kurzen Ruck anf die Seite, welche eine Positivität des Zinks anzeigt, gelst dann für cinige Secunden auf die enlgegengesetzte Seite hinüber, kehrt dann wieder auf die erste Seite zurück, verweilt hier längere oder kürzere Zeit (bei verschicdenen Versuchen wechselnd zwischen 2 und 10 Min.), kelirt dann abennals auf die entgegengesetzle Seite um, und

1) Diefs ist ganz wesentlich, weil, tvenn man die Platten zuvor cintaucht, und dann schliefst, ein Theil der Veräaderungen schon vorübergegangen ish, auf denen die folgende Erscheinung beruht. 
geht endlich 10 bis 15 Min. später (einigemale selbst noch später) nochmals auf die erste Seite zurtick, welche der Positivilät des Zinks entspricht, und von wo an ich dann weiter keine Unnkehr bcobachtet habe. In Ganzen also finden hier 5 verschiedene Phasen abwechselnd entgegengesetzter Beschaffenheit statt. Dic 3 ersten Phasen traten jedesmal auf die hier beschriebene Weise ein; statt der 4ten aber habe ich einigemale nur eine beträchtliche Schwächung der Kraft, als wollte sich die Nadel unkehren, beobachtet, wonach die Kraft wieder wuchs.

Die letzte Unkehr scheint mit einer Erschöpfung der Flüssigkeit an Silber zusammenzuhängen, was ich inzwischen uicht genau untersucht babe.

Wendet man eine mehr verdünnte Auflösung an, so dauert die erste Phase, die bei der unverdünnten Flüssigkeit blofs momentan ist, länger, und auch die spăteren Phasen erleiden Abänderung. Bei Verdünnung der Auflösung (in diesem Falle 1 Th. Salz auf $8 \mathrm{~Tb}$. Wasser) mit dem 3fachen Volume Wasser dauerte die erste positive Phase des Zinks einige Secunden, die zweite negative $1 \frac{1}{2}$ Min., in der dritten Phase ward die Nadel selir unruhig, indem sie bald gröfsere, bald klcinere Oscillationen machte, so aber, dafs doch das Zink anfangs als positiv erschien, endlich ward die Ablenkung eine Zcit lang ganz unbestimmt, indem die Nadel in grofsen Bogeu bald auf die eine, bald die andere Scite hiuuberging, bald sich in Kreise drehte; endlich aber fixirte sic sich wieder auf der Scite, welche der Positivität des Zinks entsprach, mit wachsender Kraft.

Bei Verdünnung der Auflösung mit dern 6fachen Vol. Wasser daucrte die erste positive Phase des Zinks 2 Min. lang, dann ging dic Nadel unbestimnt bold auf diese, bald anf jene Seite in grofsen Oscillationen, drehte sich mitunter ganz im Kreise, fixirte sich aber, ungefïhr $\mathbf{S}$ Min. nach der Schliefsung, auf der Seite, welche der Positivität des Zinns eutsprach, mit erst za- dann abnehmender 
Kraft. Um 14 Min. nach dem Schlusse fing die Nadel wieder an sich mehrmals im Kreise zu drehen, schwankte dann eine Zeit lang hinüber und herüber, und ging um ungefähr 17 Min. nach der Schliefsung bleibend auf die Seite über, weiche der Positivität des Zinks entsprach, mit zunehmender Kraft.

Bei Verdünnung mit dem 20fachen Voluwe Wasser blieb das Zink bleibend positiv, mindestens ward 15 Min. nach der Scbliefsung noch dieselbe Kraft als kurz nach der Schliefsung gefunden.

Durch älnliche Versuche, als ich bei einigen folgenden $\mathrm{Phänomenen} \mathrm{anführen} \mathrm{werde,} \mathrm{würde} \mathrm{sich} \mathrm{unstreitig}$ einiger Aufschlufs uber den Hergang bei dem hier angefülurten Wechsel in Richtung der Stromkraft haben erhalten lassen. Indefs ist wahrscheinlich das Phänomen ein sebr complicirtes, und es schien mir unpassend, mit Untersuchung der complicirtesten Pbänomene den Anfang zu machen, da sich uninder complicirte Fälle derselben Klasse genug darbieten. Ich habe daber die Erscheinung nicht weiter verfolgt.

Eine bestinmte Coincidenz der Wechsel in der Richtung der Stroukraft mit dem Wechsel der Niederschläge babe ich nicht darzuthun verwocht; doch ist es schwer, sicher darüber zu urtheilen. Dafs jedenfalls die crfolgenden Niederschlïge nicht gleiclıgültig sind, geht daraus hervor, dafs Verrückungen der Platten auf solche Art, dafs ihr Verhältnifs zu den Niederschlägen gestört wird, leicht neue Unkehrungen hervorbringen.

Aufser der Zinn -Zink - Kette bieten auch gar manche andere Ketten in der salpetersauren Silberauflösung noch Merkwürdigkeiten dar. Eine Kette aus Zinn und Blei z. B. verhielt sich im Moment des Eintaucheus als Zinn, Blei; die Kraft stieg, nahm dann wieder ab; 13 Min. nach der Schliefsung trat Umkehr ein (Zinn, Blei), wel- 
cher bald darauf eine Rück-Umkehrung ( $\stackrel{+}{+}$, Blei) folgte. Dieser Versuch ist blofs einmal, mit einer Auflösung, welche wie gewöhnlich t' des Salzes enthielt, angestellt worden. In gleicher Auflösung zeigte sich Platin sowohl gegen Silber als Gold positiv; wurde aber eine hinreichend verdünnte Auflüsung angewandt, so erfolgle eine allwälige Uınkehr aus resp. Silber, Platin, Gơld, Platin, in den Gegensatz.

Abwechselnde Verstärkungen und Schwächungen der Stroukraft habe ich auch noch bei verschiedeneu anderen Metallen in salpetersaurer Silberauflösuug beobachtet "); jedoch etwas genauer nur bei einer Kette von Kupfer und Eisen verfolgt, wo noch andere merkwürdige Eigenthünlichkeiten sich dawit verbinden. Hievon handelt der folgende Paragraph.

3) Wenn man Eisen mit Kupfer in starker Lösung schliefst, so verhält sich, wie bekannt, das Eisen sofort negativ gegen das Kupfer und passir. Scbliefst man beide Platten in 1 Zoll Abstand von einander, damit der Niederschlag vom Kupfer uicht zu schnell an das Eisen heranwächst, so tindet man, dafs die Kraft vom Anfange der (mit dem Eintauchen zugleich bewerkstelligten) Schliefsung erst fallt, dann wieder steigt, ein Wechsel, der sich noch mehrmals wiederholen kann, wäbrend dessen der Silberniederschlag vom Kupfer zum Eisen fortkriecht. Dieses selbst bleibt blank, und blôs ganz allmälig sieht man an detn auf den Boden aufstehenden Rande der Platte einzelne Silberflückcheu sich ansetzen. So wie das Eisen vom Silberniederschlage erreicht ist und es dadurch mit dem Kupfer verbunden wird, fängt die Kraft, die vorher stark gesticgen war, an, stark und dauernd

1) Auch in anderen chernisch differenten Flüssigkuiten sind sie nichts gar zu Seltenes, 2. B. bei verschiedenen Ketten in rauclsender Schwefelsäure, welche Flüssigkeit übcrhaupt reich an clektro-chernischen Merkwürdigkeitcu ist. 
zu fallen (unstreitig, weil jetzt ein Theil der Strömung seinen Weg durch das Silber nimmt). Der Niederschlag wächst nun bis hinter das (etwas vom Rande des kreisrunden Gefä́ses abstehende) Eisen fort, und nach längerer oder kürzerer Zeit fängt auf eimual das Eisen an, sich, von einer Stelle aus fortschreitend, zu überkupfern, und während dieses Vorgangs kehrt sich die Nadel um, worauf die Kraft sofort eine bemerkenswerthe Stärke in entgegengesetzter Richtung von vorhin erlangt, eine Kraft, die zuweilen beträchtlich gröfser ist, als diejenige, welche sie bei der ersten Scbliefsung hatte (ungeachtet der noch fortbestehenden Verbindung zwischen Eisen und Kupfer durch gefälltes Silber), die aber von da fortschreitend abnimınt, während der Kupfer-Niederschlag am Eisen wächst. Diesen Gang der Erscheinung habe ich in wiederbolten Versucben beobacbtet, und in einigen messend miltelst der Methode der Oscillationen verfolgt, indem ich die Kraft von 5 zu 5 Min. beobachtete. Bei dem einen dieser Versucbe war die zu Anfange der Schliefsung ( 0 Min.) beobachtete Kraft, mittelst der ersten 6 in merklichem Isochronisunus vor sich gebenden Schwingungen gemessen, 18,041. Sie sank von da, und bei 10 Min. ward ein Miniuum 5,4051 gefunden ${ }^{2}$ ); ron da stieg sie, bis bei 25 Min. ein Maximum 12,124 gefunden wurde; dann sank, sie wieder, bis bei 55 Min. ein Minimum 8,000 gefunden wurde, welches noch bei $65 \mathrm{Min}$. genau fortbestand, von wo an die Kraft wieder stieg, bis bei 110 Min. fast das Doppelte der vorigen Kraft, nänlich 15,688, erreicbt war, die auch bei 120 Min. noch forlbestand; darauf sank die Kraft abermals, bis bei 135 Min. ein Minimum 11,641 eintrat; stiey daun abermals

1) Während der Periode des ersten Sinkens pilegt der, die Kupferplatte anfangs wie cin Pelz überzielsende, Silbeniederschlag sich durch seine Schwere davon abzulösen, und diefs mit cinem besonderen, vom übrigen Gange der Kraft unabluängigen, Abfall derselben, der indefs raeist nicht bedcutend ist, verbunden zu sego. 
und hatte bei 148 Min. die Grölse 17,025 erreicht. Kurz darauf trat Beruhrung des Silberniederschlags mit dem Eisen ein, die Kraft sank schnell, und ungefähr um 150 Min. kehrte sich die Nadel unter Ueberkupferung des Eisens um ${ }^{1}$ ).

Als kurz (doch nicht unmittelbar) darauf die Kraft wieder gemessen wurde, fand sie sich 31,093 , sank aber von da continuirlich, und hatte unstreitio schon bei der Messung nicht mehr ganz die gleich nach der Umkehr stattfindende Grölse.

Hier folgen die Resultate eines anderen Versuchs, der uuter ziemlich ähnlichen Umständen angestellt worden ist. Ich setze von den, ehenfalls von 5 zu 5 Min. gemachten Beobachtungen blofs die Maxima und Minima ber.

$\begin{array}{rrr}0 & \text { Minuten } & 18,041 \\ 20 & - & 8,288 \\ 30 & - & 12,633 \\ 35 & - & 11,187 \\ 40 & - & 11,187 \\ 65 & - & 12,633 \\ 125 & - & 16,422 \\ 130 & - & 16,422 .\end{array}$

Zwiscben 130 und 135 Min. erreichte der Silberniederschlag die Eisenplatte.

\begin{tabular}{|c|c|c|}
\hline $135 \mathrm{M}$ & inuten & $9,590^{\text {vir }}$ \\
\hline 140 & - & 3,000 \\
\hline $143 \frac{1}{2}$ & - & $\begin{array}{l}\text { Umkehr der Nadel unter } \\
\text { Ueberkupferung. }\end{array}$ \\
\hline 45 & 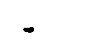 & 14,353 \\
\hline & - & 5,059 . \\
\hline
\end{tabular}

Als jetzt die Kupferplatte in der Flüssigkeit bewegt wurde, sank die Kraft fast unmerklich; als dann die Ei-

1) Diese nahe Coincidenz der Umkehr mit der Verbindung beider Platten durch Silber ist hier nur zufillig; ersterer Umstand kann viel später als letzterer eintreten. 
senplatte bewegt wurde, stieg sie sofort auf die Kraft 18,041, die beim ersten Anfang des Versuchs beobachtet wurde, und ron da an noch höher, so dafs nach einiger Zeit 35,000 beobachtet wurde.

Stellt man die Platten einander sehr nahe, etwa 1 Linie weit, in die Mitte eines Gefäfses, so erfolgt (wenigstens nach eineun Versuche) ein continuirliches Sinken der Kraft von Anfange an. Wenn der Silberniederschlag die Eisenplatte erreicht hat, so fält, wie vorhin, die Kraft noch schnell um ein Bedeutendes, und das Sinken schreitet dann langsam weiter fort, indem das Silber imner weiter hinter das Eisen forlkriechl, aber erst lange Zeit nachber erfolgt die Uıkehr der Kraft unter Ueberkupferung des Eisens.

Stellt man umgekebrt die Platten weiter von einander ( $I_{2}^{\prime}$ Zoll), so erfolgt Umkehrung mit Ueberkupferung noch bevor der Silberniederschlag das Eisen erreicht hat, und auch bier springt die Kraft plotzlich auf einen sehr hohen Werth uber. Um daher den Gang des Phänomens rein zu beobachten, wird ein weiter $\mathrm{Ab}$ stand der Platten vorzuziehen seyn.

Es hat allen Anschein, dafs die Umkehrung mit einer nahen Erschöpfung der Flussigkeit an Silber zusammenhängt, wo dann das salpetersaure Kupfer eine Veränderung anderer Art hervorbringt, als das salpetersaure Silber. In der That sab ich bei letzterem Versuche nach der Umkehr das Silber nur nocb sehr spärlich fortwachsen, und als ich nicht lange Zeit wachher den Versuch schlofs, ward. in derselben Flüssigkeit Eisen sofort positiv gegen Kupfer, und Kochsalzlösung zeigie keinen Silbergehalt melir an.

4) Der Erfolg des folgendeu Versucts liefs sich nach der Analogie der salpetersauren Silberauflösung init der Salpetersïure hinsichtlich ihrer Wirkung auf Eisen voraussehen; indessen theile ich ibn hier unit, zur Anknüpfung weiterhin folgender Versuche. 


\section{1}

Schliefst man Eisen mit Kupfer mittelst des Multiplicators in starker oder auch mä/sig verdünnter Lösunğ, so erscheint, wie auch schon in vorigen Paragraphen bemerkt, das Kupfer sofort positiv gegen das Eisen, und dieses bewirkt unter diesen Umständen keine Fällung des Silbers, wohl aber das Kupfer. Das Eisen bleibt auch dann noch negativ und passiv, wenn man allhälig so viel destillirtes Wasser zufügt, dafs frisches Eisen, mit frischem Kupfer darin geschlossen, positiv und activ wird, oder wenn man die Combination (ohne sie vorher abgewischt zu haben, wodurch die superficielle Veränderung leicht mit entfernt werden könnte) in eine gleich so verdünnte salpetersaure Silberauflösung (oder in de-, stillirtes Wasser) ubersetzt, dafs frisches Eisen mit frischem Kupfer darin positiv und activ wird ${ }^{2}$ ). Doch verdient Bemerkung, dafs nach kürzerer oder längerer Zeit durch die fortgesetzte Einwirkung der neuen Flüssigkeit die stattgehabte Veränderung allerdings aufgehoben wird, so dafs zuletzt das Eisen in die der Beschaffenheit der Flüssigkeit entsprechende Positivität und Actirität übergeht.

5) Sehr unerwarlet war mir folgende Umbehrung

1) Sollen diese Versuche gelingen und für eine wirklich stattgefundene Veränderung der Mctalle beweisen, so sind folgende liücksichten dabei zu nehmen: Die Metallplatten müssen wirtlich so weit mit der starken Lösung genetzt werden, als sie beim nachherigen Zufügen von Wasser oder Versetzen in schwächere Flüssigleit genetzt werden, damit nicht ein gewisser Antheil unveränderter Oberläche dann in Virkung komme. Beim Versetzen in Vasser oder scliwäcliere Lösung kann man den Einwand machen, dafs dic mit den Platten übergeführte Sclicht concentrirter Flüssigteit selbst eine Verstürkung der sclıwachen Flüssigkeit der Att hervorbringe, dals dic fortdauernde Negativität des Eisens dadurch bedingt werde. Inzwisetün Is̄[st sich dieser Einwurf dadurch heben, dafs man die zweite Flüssigkeit in hiareichender Menge nimmt, dic hineingesetzten Platten anfangs darin sanft hin- und herbewegt, und sich überzeugt, dafs anch noch pach dem Versuche frisches Eisen mit frischem Kupfor dorin sofort positiv und activ wird. 
des vorigen Versuchs, von deren wirklichem Stattinden ich mich inzwischen durch die vielfältigste Wiederholung überzeugt babe: Wenn Eisen mit Kupfer in salpetersaurer Silberauflüsung von solcher Verdünnung (oder auch blofsem Wasser) geschlossen wird, dafs das Eisen gegen das Kupfer sofort positiv und activ wird, so bleibt es positiv und activ, auch wenn noch allnalig so viel salpetersaure Silberlösung zugefügt wird, dafs frisches Eisen nit frischem Kupfer negativ und passiv darin wird.

6) Durch fribere Versuche ${ }^{2}$ ), die ich seitdem noch sehr crweitert babe, habe ich mich uberzeugt, dafs im Allgemeinen in den Fällen, wo zwei Metalle in einer Flüssigkeit das, dem normalen entgegengesetzte, elektromotorische Verhältnifs zeigen, sich ein Verdünnungsgrad der Flüssigkeit der Art finden läfst, dafs die Combination bei Schliefsung darin erst das normale Verhältnifs zeigt, was sich aber allmălig (oder nach Umständen nur nach einem kurzen Ruck der Nadel auf die normale Seite) in das entgegengesetzte umkehrt, indem unstreitig die, in concentrirter Flüssigkeit plötzlich erfolgende, Veränderung der Metalle, woron die Unkehrung abhängt, hier nur allmälig erfolgt. Kupfer mit Eisen in salpetersaurer Silberauflösung macht eine bemerkenswerthe Ausnabine hiervon. Wie vielfach ich auch die Verdünnung abänderte, bei keinem Verhältnisse derselben habe ich eine solche Umkehrung aus dem normalen Verbalten in das entgegengesetzte beobachten kounen; dagegen gab es ausgedehnte Verdünnungsgränzen, wo die Umkehrung in verkehrter Richtung erfolgte, indem das Eisen erst passiv und negativ gegen Kupfer war, dann, unter iminer sichtbarer werdender Schwärzung durch Fällung vou Silber, in positiv überging.

1) Dieselben sind in Schweigg. J. LIII. 61. 129. enthalten. Die sich dort vorfindende Angabe, dafs auch Kupfer nuit Eisen in rerdünnter Silberlösung das normale Umkehrungsphänomen zeige, weils ich nur durch eine zufíllige Verwechselung des Ausschlags zu crłlären. 
Ist die Flüssigkeit so weit verdünnt, dals das Eisen gleich aufangs positiv gegen Kupfer ist, so bleibt es auch positiv gegen dasselbe, ja die Stromkraft nimmt von der Schliefsung an bis zu gewissen Gränzen zu ${ }^{3}$ ). Ist andererseits die Flüssigkeit zu concentrirt, so bleibt das Eisen dauernd negaliv gegen Kupfer, abgesehen von der, im dritten Paragraphen erörterten, mit Ueberkupferung verbundenen Umkehr, welche (nach gänzlicher Veränderung der Flüssigkeit) erst sehr spät eintritt.

Die Grănzen, iunerhalb dereu das verkehrte Umkehrungspbänomen (um mich so auszudrücken) erfolgt, sind für verschiedene Sorten Eisen sehr verschieden; ja sie bleiben sich selbst für dasselbe Stück nicht immer gleich, wenn man dasselbe neu abgefeilt hat. Auch die Zeit, innerbalb deren die Umkelırung in einer gegebenen Verdünnung erfolgt, wechselt sehr, ist aber jedenfalls um so kürzer, je näber der Verdüunungsgrad derjenigen Gränze liegt, von wo an Eisen sofort und dauernd positiv gegen Kupfer ist.

Um hier einige Data mitzutheilen, so wurden bei meinen ersten Versuchen zwei Platten von derselben Eisensorte (Schleizer Eisen), welche $a$ und $b$ heifsen mögen, angewandt, deren erstere sich ein wenig negativer zeigte, als letztere. Bei 12/acher Verdüunung zeigte die Platte $a$ (nit Kupfer geschlossen) selbst nach 15 Mlin. noch keine Neigung zur Umkehr; bei $b$ erfolgte sie nach 7 Minuten. Bei 16 - bis 20facher Verdinnung erfolgte mit beiden Platten Unkelrr, in einer Zeit, die bei W'iederholung verschiedentlich ron 1 bis 9 Min. wechselte. Bei 40facher Verdunuung erfolgte bei beiden die Um-

1) Ueberhaupt sind auch bei Versıchen mit anderen Flüssigkeiten und anderen Metallen der Fälle, wo die Stromkraft vom Beginn der Sshlicliung an zoniumt, kaum weniger, als wo sie abnimmt, wofern man nur durch den Gebrauch langar Multiplicatoren den Einflufs, den die Veränderung des Uebergangswiderstandes hat, ausschliefst, und die Stromkraft vom Mnment des Fintnuelsens der Platten an verfolgt. 
kehrung binnen einigen Secunden; bei 60facher Verdünnung erfolgte mit $a$ die Umkehrung etwa nach 1 Sec., mit $b$ fand bei der Schliefsung ein momentaner Stillstand der Nadel statt, worauf sie sofort auf die, der Positivităt des Eisens entsprechende, Seile überging. Bei 80facher und 140facher Verdünnung zeigte sich $a$ resp. wach 1 Sec. oder nach einem momentanen Stillstande, $b$ sofort positiv. Bei 400facher Verdüunung waren beide sofort positiv gegen Kupfer.

Als ich diese schon vor ungefähr 2 Jahren angestellten Versuche neuerdings mit anderen Eisenplatten wiederholte, fand ich (bei zwei verschiedenen Eisensorten) die obigen Gränzen der Verdünnung für dieselben Pbänomene vicl weiler hinausgerückt, indem die eine Eisensorte selbst bei 60facher Verdünnung zuweilen noch vegativ (und zugleich passiv) blieb, die andere mindestens $\frac{1}{2}$ Minute zur Umkehr erforderte; jedoch bei Wiederbolung der Versuche mit denselben neu abgefeilten Platten blieben auch diese Bestimmungen sich nicht gleich.

7) Die nachfolgenden Versuche können, wenn nicht zur Erklärung, doch zur Erlsuterung des im vorigen $\mathrm{Pa}$ -

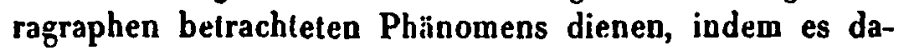
durch auf ein Spiel von Veränderungen, die ihrer Ursache und materiellen Natur nach freilich selbst unbekannter Beschaffenheit sind, zurückgeführt wird. Das Resultat, was ich voranstelle, ist folgendes:

An dem ganzen Phänomen haben Veränderungen des Eisens den Hauptantheil, obschon das Kupfer nicht ganz frei davon bleibt. In starker Flüssigkeil erfährt, wie schon früher bekannt, das Eisen eine sofortige und bleibende Veränderung, die es elektro-negativer macht, und bleibt blank (passiv); in sehr verdünnter Flüssigkeit erfährt es eine solortige und bleibende Veränderung von positiver Natur, und scbwärzt sich sofort beim Eintauchen; innerhalb der Verdünnungsgränzen, wo das Umkehrungsphänomen eintritt, erfährt es sofort beim Ein- 
tauchen eine Veränderung von negativer Natur und ist anfangs blank; bald aber schwärzt es sich, gewöhnlich von einzelnen Stellen aus fortschreitend, und nie erfolgt die Umkehr eher, als wenn dic Schwärzung eine gewisse Ausdehnung erlangt hat, womit dann zugleich die negative Veränderung in eine nachweisbar positive übergegangen ist. Das Kupfer verhalt sich in starker wie in verdünnter Flüssigkeit wie ein negativ verändertes $\mathrm{Me}$ tall, aber seine Veränderung kommt nicht in Betracht gegen die des Eisens.

Hier folgen die, in ihrem Detail noch manche interessante Punkte darbietenden, Versuche.

a) Wenn man Eisen, was in starker Flüssigkeit steht, mit dem vorragenden Ende an einem selbst eisernen Condensator anbringt, wïhrend die Flüssigkeit durch einen Streifen destillirten Wassers mit dem Erdboden communicirt, so wird der Condensalor stark positiv geladen, was nicht anders seyn kann, wenn das in der Flussigkeit stebende Ende wirklich negativ verändert worden ist. Weun man dagegen mit Eisen in stark verdüunter Flüssigkeit (worin es sich schwărzt) denselben Versuch anstellt, so wird der Condensator stark positiv geladen. Manchual findet man, dals ein Stück Eisen den Condensator positiv ladet, während ein anderes in derselben Flüssigkeit stchendes ihn negativ ladet, und dann findet sich ohne Ausnahme, dafs ersteres sich geschwärzt bat, während letzteres blank geblieben ist; eine Coincidenz der Zustände, die ich durch die vielfachsten Abänderungen mit überraschend constanten und augenfälligen Resultaten verfolgt habe. Wendet man eine Flüssigkeit ron solcher Verdüunung an, dafs ein gegebenes Stück Eisen erst nach einiger Zeit sich darin schwärzt und mit Kupfer das Unkehrungsphänomen zeigt, so findet man durchaus constant, dafs, so lange die Schwärzung noch nicht einige Ausdelinung erreicht hat, der Condensator positiv, nachher aber durch dasselbe Stück negativ geladen wird. 
Dasselbe Pbänomen des successiven Wechsels der Ausschläge kann man auch in einer schr verdünnten Flüssigkeit, die unter den gewöhnlichen Umständen sofortige Sclıwärzung und Positivität des Eisens bervorbringt, dadurcb erzeugen, dafs man das Eisen erst in die starke Flüssigkeit eintaucht, dann mit der anhängenden Flüssigkeit in die verdünnte Losung versetzt. Unter solchen Umständen dauert es oft ziemlich lange Zeit, dafs das Eisen blank bleibt, und eben so lange ladet es auch den Condensator positiv; diefs findet auch (eben so in den vorigen Fällen) noch dann statt, wenn die Schwärzung schon begonnen hat; aber noch ehe die ganze Platte davon ergriffen ist, tritt schon ein lebbafter entgegengesetzter Ausschlag ein.

Einigemale habe ich, wenn die Schwärzung schon begonnen batte, jedoch das Eisen den Condensator noch positiv lud, die scheinbar blanken und passiven Stellen der Oberfläche mit einem höchst zarten Flaum der feinsten metallglänzenden Silbernadeln überzogen gefunden, der jedoch nur gut sichtbar bleibt, so lange man das Eisen in seinen Verhältnissen zur Flüssigkeit nicltt stört, dagegen beim Herausziehen sich in einen feinen Schlamen verwandelt. Vielleicht sind die Anfănge dieses krystallinischen Niederschlags selbst schon vor begonnever Schwärzung vorbanden. Inzwischen habe ich anderemale nichts von diesem Phänomen wahrnehmen können.

b) Taucht man zu Eisen, was schon eine Zeit lang in salpetersaurer Silberaullösung gestanden hat, frisches Eisen, so verhält sich das erst eingetauchte Metall stets positiv gegen das hinzugetauchte, wenn jenes activ darin ist, negativ hingegen, wenn es passiv darin ist. Innerhalb der Grănzen des Umkehrungsphänomens kann man daher beliebig einen Ausschlag des Multiplicators nach der einen oder anderen Seite erhalten, je nachdem man das zweite Eisen in der ersten oder zweiten Periode hinzutaucht. 
c) Schliefst man Kupfer mit Eisen in solcher Verdünnung, dafs das Eisen sich gleich anfangs schwärzt, so erfolgt (wie bei verschiedenen Verdünnungen beobachtet wurde) gleich rom Anfange an ein Steigen der Kraft, was erst spät in ein Sinken übergeht. . Z. B. in 384 facher Verdünnung wurde folgender Gang der successiven Oscillationen beobachtet: $25 ; 24,5 ; 24,5 ; 24$; 23,5; un 5 Min. nach der Schliefsung 21,5; um 1 Stunde 20; um 21: Stunde 21,5.

d) Taucht man innerhalb der Verdünnungsgränzen des Umkehrungsphänomens das Eisen eine Zeit lang vor dem Kupfer ein, so wird, wenn nach erfolgter Schwärzung das Kupfer zugetaucht wird, das Umkehrungsphänomen nicht erfolgen, sondern das Eisen sich sofort und dauernd positiv gegen das Kupfer zeigen. Taucht man dagegen das Kupfer eine gleich lange Zeit vor dem Eisen, so wird beim nachherigen Hinzutauchen des Eisens das Unkehrungsphänomen wie gewöhnlich eintreten.

e) Das Kupfer theilt, in starker Lösung stehend, einem kupfernen Condensator positive Elektricität mit, was für eine negative Veränderung des in der Flüssigkeit stebenden Theils spricht. Bei Verdünnungen, innerhalb deren das Unkehrungsphänomen erfolgt, erhält man keine deutlichen Resultate mebr am Condensator.

f) Stellt man inzwischen in eine Flüssigkeit von solcher Verdünnung ( 48 fache) eine Kupferplatte, und gleichzeitig eine zweite in ein anderes Gefäfs mit destillirtem Wasser, verbindet man dann die Platten durch den Multiplicator, die Flüssigkeit der Gefärse aber durch eine, in feine Oeffnungen endigende, gebogene Röhre, welche selbst mit destillirtem Wasser gefüllt ist, so zeigt der Multiplicator die Negativität des in der Silberlosung stehendeq Kupfers an.

g) Stellt man Versuche mit ungleichzeitigem Eintauchen homogener Platten an (bei 1 bis 5 Min. $Z$ wischenzeit), so zeigt sich das erst eingetauchte Kupfer positiv

Poggendorff's Annal. Bd, XXXXVII. 
gegen das hinzugetauchte, mag die Flüssigkeit stark oder bis innerhalb der Gränzen des Uınkehrungsphänomens verdünnt seyn. Der Ausschlaz uach dem hinzutauchen kehrt sich aber immer sehr bald (noch vor 1 Min.) un, mag das Zutauchen in der kürzeren oder längeren Zwischenzeit erfolgen.

h) Combinirt man das Resultat der Versuche $e$ und $f$ mit $g$, so mufs man annehmen, dafs die negative Veränderung des Kupfers gleich bein Eintauchen (wo es sich in der That sofort schwärzt) im Maxinum ist, und später wieder abnimmt '). Als einen unterstützenden Bewcis für diese Annahue kann man den Umstand ansehen, dafs, wenn man eine Platinplatte längere Zeit in einer mäfsig (32- bis $48 \mathrm{fach}$ ) verdünnten Auflüsung hat stehen lassen und dann Kupfer mit Schliefsung binzutaucht, der Strom eine vom Anfange an wachsende Kraft zeigt.

i) Mag mau das wachsende Metall nach 1 Min. oder nach 5 Min. zutauchen, so erscheint doch innerbalb der Gränzen des Unkehrungspbünomens ( 32 - bis 48 facler Verdünnung) die Positivität des erst eingetauchten Metalls beträchllich grüfser bei Eisen, als bei Kupfer. So z. B. wurden für Eisen bei zwei Versuchen mit verwechselten Platten die Oscillationzahlen 16 und 15,5 für die Aufangskräfte erhalten, für Kupfer dagegen, unter vergleichbaren Uinstanden 74 und 71 . Bemerkung indefs verdient, dafs bei diesen Versuchen mit dem Kupfer schon während der ersten zwei Oscillationen eine Verzögerung

1) Dieser Fall konmt ofters vor; besonders auffallend in Schwrefelleberlösung bei Kupfer, Wismuth, Platin und anderen Metallen, wo jedoch die Veränderung positiver Natur ist. Es scheinen mir solche Fälle darauf zu beruhen, dals in manchen Flüssigkeiten zwei Veränderungen entgegengesetzter Art, vielleicht durch die verschiedenen Bestandtheile der Flüssigkeit, erfolgen, von denen die eine schnell auf dem Maximum, und überwiegend ist, die andere langsamer fortschreitend jene mindert, wohl selbst bis zur Umkehr, letzteres z. B. bei Zint in Schwefelleberlösung. 
sehr sichtbar ist, daher diese Zahlen allerdings nicht zum reinen Maafse der anfänglichen Stromkraft dienen können.

$k$ ) Es gelang mir, zwei Eisensorten zu finden, von denen die eine in 24 facher Verdünnung sofort sich schwärzte, die andere in derselben Verdünnung dauernd blank blieb. Jene gab bei Schliefsung mit Platin, was schon lingere Zeit (10 Minuten) in dieser Verdünnung gestanden hatle, folgende successive Anfangs - Oscillationszablen: 18, 16, 15; diese aber 57, 60,63 und acht Min. nach der Schliefsung 72. Also bei dem ersten Eisen nahm die Kraft zu, indem das Eisen positiver wurde, bei dem letzten nabm sie ab, indem die Negativität zunahm; und dic Anfangskräfte verhielten sich, wenn man von der, warscheinlich schon während der ersten 2 wei Oscillationen merklichen, Aenderung absiebt, wie 25,I zu 1,62. Gewvifs ein merkwürdiges Resultat. In einer 32 fachen Verdünnung zeigte ein sich unmittelbar schwärzendes Eisen, mit dem lange gestandenen Platin geschlossen, folgende Succession der Oscillationszeiten: 21, 19, $17,16,16,15,15$.

Schliefst man Kupfer unmittelbar beim Eintauchen mit Platin, was schon 10 Min. in der Flüssigk eit gestanden hatte, so zejgt sich bei verschiedenen Verdinnungen eine vom Anfange an schwach und langsan steigende Kraft, 2. B. in 32 facher Verdünnung folgende Oscillationszeiten: 27, 26, 25, 25, 25; auch 5 Min. naciher noch 25. In 24 facher Verdünnung: $27,25,24,5,24,5$, 24, 24, 24. Sebr weit habe ich den Gang der Wirkungsäuderung mit Fleifs hiebei nicht verfolgt; weil Platin, was 10 Min. in salpetersaurer SilberIósung gestanden hat, selbst noch eine langsam fortschreitende Aenderung erfährt, wie weiterhin folgende Versuche (die sich allerdings auf starke Lösung beziehen) zeigen werden.

l) Läfst man ein, sich in ciner gegebenen Verdünnung sofort schwärzendes Eisen erst längere Zeit in der Flüssigkeit stehen, ehe man es mit dem Platin, was schon 
längere Zeit in derselben Flüssigk eit gestanden bat, schliefst, so erhält man eine stärkerc Anfangskraft, als wenn man es uumittelbar beim Zutauchen mit den Platin schliefst, was beweist, dafs das unter $k$ bemerkte Steigen der Stromkraft abhängt von einer Aenderung, die nicht selbst erst durch die Schliefsung hervorgerufen wird.

In Betreff der vorigen Versuche bemerke ich noch im Allgemeinen, dafs nach jedem Versuche die Flüssigkeit, in welcher eine Fällung darch Eisen oder Kupfer stattgefunden hatte, weggeworfen wurde, um nicht bei neıen Versuchen mit einer veränderten Flüssigkeit $\mathbf{z n}$ thun zu haben, dafs jedoch mit Fleils auch einige Versuche in derselben Flüssigkeit wiederholt worden sind, welche schon zu einein vorherigen Versuche gleicher Art gedient hatte, um durch das Constante der Resultate den Beweis zu erhalten, dafs die angezeigten Wirkungsänderungen der Kette nicht von einer Aenderung der Flüssigkeit abhängen.

8) Ungeachtet ich eine sehr grofse Anzahl Metalle und Flüssigkeiten hinsichtlich ibrer Umkehrungsphänomene und des Verhaltens, was sie bei ungleicbzeitigem Eintauchen darbieten, geprüft habe, ist mir docb eine solche Umkehrung in der, der vormalen entgegengesetzten Richtung, als vorhin beschrieben worden, sunst blofs noch bei einigen Metallen in gesättigter oder wenig verdünnter Schwefelleberlüsung rorgekommen. Hier nämlich zeigen die Combinationen Zinn, Kupfer, Eisen, Wismuth ebenfalls dic Eigenthümlichkeit, aus -+ in +in kurzer Zeit überzugehen.

Bei Zinn-Kupfer bin ich den biebei stattindenden Veränderungen speciell nachgegangen, und es hat sich hieraus ergeben, $a$ ) dafs sowobl Zinn als Kupfer durch Schwefelleberlösung positiv verändert werden, $b$ ) dals die anfängliche Veränderuug des Kupfers viel stïrker ist, als die des Zinns, aber schnell in so starken Verhăltnisse abuimmt, dafs das Kupfer dadurch bald wieder un- 
ter das Zinn gerückt wird. Dic Verhältnisse der verschiedenen Metalle in Schwefelleberlösung sind überhaupt so interessant, dafs sie eine besondere Betrachtung verdienen. Ich begntige wich daher hier wit dieser vorläufigen Anzeige.

9) Gewöhnlich pllegt man anzunehmen, dafs Platin ein, durch. fast alle Flüssigkeiten unveränderliches Metall sey, und an wenigsten dürte man in der salpetersauren Silberlösung das Vernögen suchen, eive denderung daran hervorzubringen. Inzwischen, wie man auch die nachfolgenden Erfahrungeu deuten möge, gewiifs ist, dal's das in diese Lösung eingetauchte Platin bald vach dem Eintauchen ein ganz anderes elektrisches Vernögen zeigt, als späterbiu. Im Sinne meiner Ansicht würde ich das Resultat so ausdrücken: Das Platin erfälut im Moment seines Eintauchens eine Aenderung, durch die es beträchtlich an Negativität zunimmt; allein diese Negativität unindert sich fortgehends mit der Zeit ' ). So unwahrscheinlich diese Annabme erscheinen mag, so weils ich doch den naclfolgenden Thatsachen keine andere Deutung zu geben; und gewifs ist wenigstens, dafs alle

1) In meiner Abbandlung in diesen Annalen, Bd. XXXXII S. 508, hatte ich aus dem positiven Verhalten des Platins gegen Gold in salpetersaurer Silberlösung, so wie daraus, dals erst eingetauchtes Platin sich positiv gegen später zugetauchtes in dieser Lösung verhälk, geschlossen, dafs die Veränderung des Platins in diester Flüssigkeit positiver Natur sey. Inzwischen kann man aus ersterem Umstande keinen Beweis ziehen, so lange nicht die Veränderungen des Goldes in dieser Flüssigkeit für sich untersucht sind; und letzterer Umstand erkJärt sich im Zusammenhange mit den übrigen, im Folgenden mitzutheilenden Versuchen richtiger durch die Abnahıme der anfãuglichen negativen Veränderung. Wenn übrigens in dieser Abhandlung fast blofs von Aenderungen die Rede ist, die sich vom Infange an mindern, so darf man hierin nicht etwas Allgemeines suchen. Vielmebr kann man, namentich in nicht zu concentrirten Flüssigkeiten, in unzäbligen Fällen das Wachsen der Veränderungen in der anfänglichen Richtung durch ähaliche als obige Versuche selir gut verfolgen. 


\section{2}

übrigen Erklärungsweisen, die man bei solchen Fällen anzuwenden pllegt, eben so viel dabei unerklärt lassen.

a) Wenn man Platin, in starker salpetersaurer Silberlösung stehend, mit seinem vorragenden Ende an dem kupfernen oder silbernen Condensator mit Hülfe eines feuchten Zwischenblattchens entladet (wishrend auch die obere Condensatorplatte durch ein solches Blättchen mit dem Boden communicirt), so wird der Condensator lebhaft positiv geladen, was eine negative Aenderung des in der Flüssigkeit, befindlichen Theils voraussetzt. (Eben so verhält sich Silber bei directer Anbringung am silbernen Condensator). Auch in 32 facher Verdiunnung war das angegebene Verbalteu des Platins noch sebr merkbar.

b) Wenn man zwei Platinplatten, die eine in ein Gefäfs mit destillirtem Wasser, die andere in ein Gefäls mit starker salpetersaurer Silberlösung stellt, welche durch einen, halb mit Silberauflösung, halb mit destillirtem Wasser genetzten Papierstreifen communiciren, so verhält sich das Platin in der Silberauflösung als das negative Metall. Es bestătigt sich diefs, auch wenn man die Platten, neu gereinigt, wechselt, was uberbaupt nie bei Versuchen dieser Art zu übergehen, damit nicht ein ursprünglicher Mangel an Homogenität der Metalle EinAlufs gewinne. Die Reinigung des Platins geschah hier, wie bei allen Versuchen dieser Abbandlung, stets so, dafs es erst abgetrocknet, dann mit Sandpapier (Glaspapier, Rostpapier), was mit destillirtem Wasser genetzt war, dann noch mit trocknem Sandpapier bestens abgescheuert wurde.

c) Läfst man eine Platinplatte kürzere oder längere Zeit (1 Min. bis mehre Stunden) in starker Lösung eingetaucht, und taucht dann eine $\mathbf{z w e i t e ~ h i n z u , ~ s o ~ v e r b a ̈ l t ~}$ sich die erste stets positiv, was entweder auf eine wachsende positive oder eine abnehmende negative Aenderung des Platins in der Flüssigk eit deutet. Die vorigen 


\section{3}

Versuche haben die Negativităt der Aenderung gezeigt, die folgenden werden ibre Abnabme dartbun.

d) Man tauche eine Platinplatte und eine Eisenplatte in starke Lösung gleichzeitig mit Schliefsung ein, und beobachte die Anfangskraft durch die Methode der Oscillationen oder der Ablenkungen. Man wird finden, dafs diese Kraft rom Anfange an rasch abninmt. Dafs nun diese Abnahme nicht von der Schliefsung abbängig sey, ergiebt sich aus den folgenden Versuchen.

$e$ ) Hat man das Platin längere Zeit in der Flüssigkeit stehen lassen, ehe man das Eisen mit Schliefsung dazu taucht, so ist die Anfangskraft gleich anfangs beträchtlich geringer, als bei gleichzeitigew Eintauchen und Scbliefsen, die Abnahme aber langsamer. Hier von mehreren Wiederholungen einige Beispiele:

Bei gleichzeitigem Eintauchen und Schliefsen ron Eisen und Platin waren Folgendes die Zeiten der successiven zwei Oscillationen vom Anfange an:

$$
57,61,64,5,68,70,72 .
$$

Als das Platin schon 5 Minuten in der Fllissigkeit gestanden, ehe das Eisen mit Schliefsung dazu getaucht ward:

$$
72,73,73,74 .
$$

Als das Platin 13 Minuten in der Flüssigkeit gestanden, ehe das Eisen dazu geschlossen ward:

$79,79$.

Um jeden Verdacht zu begegnen, dafs die vorgängige Schliefsung bei einem früheren dieser Versuche auf das Resultat des späteren Versuches influirt hätte, war das Platin nicht (um etwa Zeit zu sparen) von einem dieser Versuche zum andern in der Flüssigkeit gelassen, sondern nach jedem Versuche herausgezogen, nen auf angegebene Art gereinigt und die angegebene Zeit abgewartet worden, bevor das frisch abgefeilte Eisen zugetaucht wurde. 
Bei einer anderen Versuchsreihe mit anderen Eisenund Platin-Platten war die Zeit der zwei Aufangs-Oscillationen, wenn beide Platten zugleich mit Schliefsung eingetaucht wurden, 52, wiederum mit folgender rascher Verlangsamung. Wenn aber das Platin schon 20 Min. in der Flüssigkeit gestanden hatte, ehe das Eisen mit Schliefsung dazu getaucht wurde, 81, wonach auch die noch beobachteten folgenden vier Oscillationen zu je 2 in 81 Zeittheilen vollbracht wurden.

Bei noch einer anderen Versuchsreihe, wo statt Oscillationen Ablenkungen angewandt wurden, betrug die erste Ausweichung, wenn Platin und Eisen zugleich mit Schlicfsung eingetaucht wurden: $163^{\circ}$ mit dem Rückgang auf $8^{\circ}$ derselben Seite. War aber das Platin 5 Min. eingetaucht, ehe das Eisen wit Schliefsung dazu getaucht ward, blofs $82^{\circ}$ mit dem Rückgang auf $20^{\circ}$.

Noch ein anderes Mal ward die Kraft beobachtet, welche 5 Minuten nach gleichzeitigem Eintauchen und Schliefsen beider Platten stattfand, und beträchtlich grofser gefunden, als die Anfangskraft, welche sich zeigte, wenn das Eisen zum Platin mit Schliefsung getaucht ward, nachdem letzteres schon eine halbe Stunde in der Flüssigkeit gestanden.

Lälst man Eisen und Platin, beide längere Zeit in der Flüssigkeit stehen, bevor man sie schliefst, so findet man eben so eine sehr geringe Anfangskraft, als wenn man das Platin allein lăngere Zeit darin hätte stehen lassen, und diese geringe Kraft bleibt wenigstens mebrere Oscillationen durch (so lange habe ich sie nur verfolgt) coustant. Hat man dagegen das Eisen allein lange Zeit in der Flüssigkeit stehen lassen, ehe man das Platin mit Schliefsung dazu taucht, so ist die Anfangskraft, durch die zwei ersten Oscillationen gemessen, so wenig verschieden von der, welche man erbält, wenn man Platin and Eisen gleichzeitig mit Schliefsung eintaucht, dafs die Verschiedenheit inuerhalb der engen Gräuzen fällt, wel- 
che Wiederholung selbst derselben Versuche nach neuer Reinigung der Platten darbietet, und die Kraft mindert sich ebenfalls rasch.

$f)$ Hat man Platin mit Eisen längere Zeit in starker Lösung geschlossen stehen lassen, so dafs die Kraft nur noch sehr gering 1st, so erfolgt keine in Betracht kommende Wiederherstellung der Kraft, wenn man die Eisenplatte in der Flüssigkeit bewegt, wofern nur möglichst ein Anwogen an der Platinplatte vermieden wird; eben so wenig, wenn man die Eisenplatte herauszieht, und nach neuem Abfeilen wieder eintaucht '); dagegen starke Bewegung der Platinplatte in der Flüssigkeit immer eine partielle, und Herausziehen und Erneuung der Oberfläche ungefähr die volle Wiederherstellung der ursprünglichen Kraft mit sich bringt. (Kleine Unterschiede bei Wiederholung desselben Versucbs erlauben nicht, das Resultat ganz scharf auszusprechen.) Zieht wan die Platioplatte heraus, spült sie in destillirtem Wasser ab, trocknet sie über heifsen Kohlen und taucht sie nach dem Erkalten wieder zum Eisen hinza, so verbält sie sich sogar positiver als das Eisen. Doch ist letzterer Versuch nur ein Mal angestellt.

10) Die Veränderung, welche die salpetersaure Silberlösung ain Platin hervorbringt, ist keinesweges eine besondere Eigenthümlichkeit dieser Flüssigkeit; vielmehr zeigt sich durch ähnliche Versuche, als die angeführten, das Platin uberbaupt durch die verschiedensten Flussigkeiten elektro-chemischer Veränderungen fähig, die oft sehr auffallend sind; und ganz besonders stark ist diejenige, welche es durch Salpetersäure erleidet, die man gewöhnlich als ein Reinigungsmittel des Platins ansieht. Blofs beiläulig und andeutungsweise, da diese Abhand-

1) Eine unbedeutende, schnell wieder verschwindende, Erhöhung bemerkte ich allerdings öfters, andere Male indefs such wieder das Gugentheil. 
lung nicht bestimmt ist, den Gegenstand auszufuhren, mögen folgende Versuche darüber hier Platz finden.

Eine Platinplatte $a$ wurde 4 Minuten in rectificirter Salpetersăure ron 1,280 spec. Gew. stehen gelassen, dann herausgenommen, mit weifsem Löschpapier erst abgetrocknet, dann auf das Stärkste mit trocknem Löschpapier abgerieben. Darauf ward sie mit einer anderen, soust ihr hamogenen Platinplatte $b$, deren Oberfläche durch nasses und trocknes Reiben mit Sandpapier völlig erneuert war, durch gleichzeitiges Eintauchen in Kochsalzlüsung mittelst neines längsten Multiplicators geschlossen. Es erfolgte ein Ausschlag von $+147^{\circ}, 5$, welcher die Negativität der mit Salpetersăure behandelt gewesenen Platte anzeigte. Die Gränze des Rückganges der Nadel war $-5^{\circ}, 5$. Der Versuch ward jetzt so wicderholt, dafs die Platte $a$, nachdem sie neu mit Sandpapier gescheuert, wieder 4 Minuten mit der Salpetersäure in Berührung gelassen, dann abgetrocknet, und stark mit Löschpapier abgerieben worden war, noch uberdiefs 1 Minute lang in destillirtem Wasser hin - und herbewegt, und dann abermals mit Löschpapier getrocknet und stark abgerieben wurde, bevor sie mit der, auf's Neue mit Sandpapier abgescheuerten, Platte $b$ in Kochsalzlösung gescblossen wurde. Die Gränzen des ersten Hin- und Rückganges waren jetzt $+145^{0}, 5 ;-41^{0}, 5$; so dafs wiederum $a$ als negativ erschien. Beide Platten wurden jetzt mit destillirlew Wasser gespült, abgetrocknet, ihre Oberfläche durch sorgfältigstes Abscheuern mit Sandpapier erneuert, und daun der vorize Versuch mit seiner ganzen Procedur des 4 Minuten langen Eintauchens u. s. f. wicderholt, aber mit verwechselten Platten, um jeden Verdacht auszuschliefsen, dafs Mangel an eigentbümlicher Homogenität der Platten den Eriolg bedingt babe; der Ausschlag zu Gunsten der Negativität der Platte $b$ war jetzt $+157,5$, mit dem Rückgang auf $-2 \overline{7}, 5$.

Un endlich für beide Platten alles möglichst gleich 
zu machen, habe ich zu einer ganz anderen Zeit den Versuch in Salpetersăure von derselben Beschaffenheit, blofs mit folgenden Abänderungen wiederbolt. Dic eine Platte blieb blors 3 Minuten in der Säure eingetaucht, sie wurde nachber, wie oben, abgetrocknet, stark mit Loschpapier abgerieben und zugleich mit der andern, mit Sandpapier zuvor gereinigten, Platte 1 Minute lang in destillirtem Wasser bewegt, dann beide abgetrocknet und in Kochsalzlösung geschlossen. Der Ausschlag zu Gunsten der mit Salpetersäure behandelten Platte war das eine Mal + 122 mit dem Rückgang auf - 106; und noch 7 bis 8 Minuten nachber betrug die Ablenkung ungefähr $6^{\circ}$. Bei Wiederbolung des Versuchs mit verwechselten (zuvor neu gereinigten) Platten schlug die Nadel wieder zu Gunsten der Negativität der mit Salpetersäure behandelten Platte so weit aus, dafs sie sich im Kreise nach dieser Richtung ${ }^{\circ}$ fortdrehte, ungeachtet sie vorher merklich still gestanden; ein Umstand, den ich nicht zu erklären weifs, da $180^{\circ}$ das Maximun des ersten Ausschlags scheint seyn zu müssen. Um 5 Minuten nach der Schliefsung stand die Nadel noch auf $7^{\circ}, \mathbf{5}$.

$\mathrm{Zu}$ diesen Resultaten füge man folgende: $\mathrm{Das} \mathrm{Pla}$ tin in derselben rectificirten Salpetersäure theilt dem Condensator sebr lebhafte positive Elektricität mit, was für die negative Veränderung des in der Flüssigkeit stehenden Theils spricht. Herausgenommen, vollkommen mit Losschpapier abgetrocknet, und an dem Theile gefafst, welcher mit der Säure in Berübrung war, während der andere am Condensator angebracht wird, entstelt immer noch ein ziemlich lebhafter positiver $\Lambda$ usschlag. Selbst dann ist dieser noch deutlich genug, obschon an Stärke sehr vermindert, wenn man die genetzt gewesene Stelle wit den Losschpapier so stark als möglich abgerieben. Spült man aber die Stelle in destillirtem Wasser ab, so zeigt der Condensalor nichts webr deutlich. Indefs bat uns der viel empfindlichere Multiplicator gelehrt, dafs 
selbst jetzt die Veränderung noch vicht vollståndig aufgehoben ist. - Auch in rectificirter Salzsäure (von 1,140 spec. Gew.) und rectificirter Schwefelsăure (von 1,580) ladete das Platin den Condensator positiv, und nach Herausnahme und Abtrocknen bestand dieser Ausschlag ebenfalls fort, wenn das Platin an der genetzt gewesenen Stelle gefafst wurde.

Versuche mit ungleichzeitigem Eintauchen beweisen ebenfalls, wie verschieden das Platin durch verschiedene Flüssigkeiten afficirt werden kann. So fand ich bei 3 Minuten Zwischenzeit des Eintauchens stets das erst eingetauchte Platin negativ gegen das zweite in den genannten drei rectificirten, so wie auch noch stärkeren käuflichen Säuren, in Aetzkalilosung ( $1 \mathrm{Th}$. in $3 \mathrm{Th}$. Wasser), in Kocbsalzlösung, Bleizuckerlösung, Brunnenwasser; dagegen positiv in salpetersaurer Silberlösung, Schwefelleberlösung, wäfsriger schwefliger Săure, Kupfervitriollösung.

Der erste Ausschlag in der rectificirten Salzsăure betring (im Mittel zweier Versuche mit verwechselten Platten) $+21^{\circ}, 5$ mit dem Rückgang auf $-3^{\circ}$; in der rectificirten Schwefelsäure $+83^{\circ}$, unit dem Rückgang auf $-38^{\circ}$; in der rectificirten Salpetersäure bei zuerst eingetauchter Platte $a+148^{\circ}, \overline{5}$ mit dem Rückgang auf $-87^{\circ}, 5$. Bei zuerst eingetauchter Platte $b$ drehte sich die Nadel beim ersten Ausschlage sogar wieder im Kreise.

Man wird nach so auffallenden Beispielen ron Veränderungen, welche die Metalle durch stark angreifende Flüssigkeiten crfabren, unstreitig zugeben, dafs Veränderungen dieser Art bei der berühmten Becquerel'schen Kette nicht cinflufslos scyn können. Die Wirksamkeit dieser Kette wird gewöbnlich von der Wirkung des Kali auf die Salpetersäure abgeleitet, und es ist unzweifelhaft, wie ich mich, frühere Versuche von No bili und B e cquerel bestätigend, überzeugt habc, dafs, unabbängig von aller Berülrung mit Metallen, hiedurch wirklich ein Strom 
entstelit. Allein eben so entscheidend lafst sich darthun, dafs es bei der B ecquerel'schen Anordnung in der That uicht dieser Strom ist, sondern der durch die Berubrung and, wie ich glaube, Veränderung der Metalle von Seiten der heterogenen Flüssigkeiten erweckt wird, von welchem die Hauptwirkung ausgeht. Es ist indefs hier nicht der Ort, ausführlich davon zu handeln; ich kehre vielmebr nach dieser Abschweifung zur salpetersauren Silbcrlösung zurück.

11) Der negative Zustand, welchen das Eisen unmittelbar beim Eintauchen in starker Lösung erbält, ändert sich nachher nur noch wenig; indefs lehren die Versuche, dafs er einige Momente nach dem Eintauchen noch zunimmt, nachher allwălig und sehr wenig abnimmt.

Mit Oscillationsversuchen kann man diese Uınstände nicht nachweisen, weil schon während der ersten zwei Oscillationen die Abnahme wieder beginnt, auch die ganze Kraft zu schwach ist. Indefs geht die angegebene Thatsache aus folgenden Umständen hervor.

Ungeachtet die bedeutende Negativität des Eisens in salpetersaurer Silberlösung durch Versuche mit dem Condensator und auf andere Weise zur Genüge dargethan wird, so erbält man doch, wenn man Eisen in starke Losung eintaucht und später Eisen dazu schliefst, immer nur einen geringen dusschlag durch das ungleichzeitige Eintauchen, in welcher Zwischenzeit (15 Secunden bis 21 Stunden) nach der ersten auch die zweite Platte eingetaucht werden mag; ein Beweis, dafs die zweite Platte im Moment des Eintauchens schon fast eben so stark verăndert wird, als die erste. Inzwischen zeigt dieser Ausschlag stets eine Negativität der erst eingetauchten Platte an, also kann die Negativität im Moment des Eintauchens doch nicht auf das volle Maximum kommen. Hat man die erste Platte lange Zeit (bei einem Versuche 21 Stunden, bei einem auderen 25 Minuten, bei ei- 
nem dritten mit verwechselten Platten 53 Min.) eingetancht gelassen, ehe man die zweite dazu taucht, so erfolgt nach einer Bewegung von wenigen Graden, welche der Negativität der erst eingetauchten Platte entspricht, oder auch nur einem kleinen Ruck auf diese Seite, sofort eine Uınkehrung der Nadel auf die entgegengesetzte Seite, und die Kraft lährt dann fort nach dieser Seite zu wachsen (was ich respectiv durch 5 bis 15 Min. bei verschiedenen Versuchen verfolgt habe). Lälst man die erste Platte kürzere Zeit eingetaucht, bevor man die zweite Platte dazu taucht, so findet man bei vergleichenden Versuchen wit jedesmal erneuerter Oberläche der Platten, dafs der erste Ausschlag, obwohl immer niclt bedeutend, doch um so beträ́chtlicher ist, je früher (bis zu $\frac{1}{4}$ Min.) nach der ersten Platte man die zweite hinzutaucbt. So betrug er, bei einer Zwischenzeit von $\frac{1}{4}$ Min. $15^{\circ} \frac{1}{2}$, bei 1 Min. $9^{\circ} \frac{1}{2}$, bei 2 Min. $7^{\circ} \frac{1}{2}$, bei 53 Min. noch keinen ganzen Grad, wonach sofort Umkehrung der Nadel erfolgte. Bei Wiederbolung mit verwechselten Platten betrug der erste Ausschlag bei einer Zwischenzeit von 21 Stunden $3^{\circ}$, wonach sofort Umkehrung eintrat. Diese Versuche sind inzwischen blofs wit zwei Platten angestellt, und es wäre möglich, dafs bei manchen Eisensorten eine längere Zwischenzeit des Eintauchens oder etwas andere Beschaffenheit im Neutralitätszustande der Lösung sogleich einen positiven Ausschlag für die erst eingetauchte Platte mit sich brächte. In der That kann man nicht vorsichtig genug seyn, Resultate dieser Art zu verallgemeinern, bevor man sie unter mehrfachen Umständen geprüft. Stellt man Versuche dieser Art in Verdünnungen an, in welchen sich das Eisen nicht schwärzt, so findet man, dafs für gleiche $\mathrm{Zwischenzeil} \mathrm{des} \mathrm{Eintau-}$ chens ein viel stärkerer anfänglicher Ausschlag in der verdünnten, als in der starken Lösung erhalten wird, was bestätigend für den Umstand ist, dafs in starker Lösung 
die Veränderung sehr schnell erfolgt. Der Versuch wurde bei 1 bis 3 Min. Zwischenzeit in 32 facher Verdünnung mit denselben (hier blank bleibenden) Platten, als in der starken Lösung angestellt.

Verbindet man ein, mit starker Lösung, und ein, mit destillirtem Wasser gefülltes, mit einem Papierstreifen, der halb mit jener Lösung, balb mit Wasser genetzt ist, und tauclit in das eine Gefăfs eine Eisenplatte, in das andere eine Platinplatte, so ist immer das Metall negativ, welches in der Silberlösung stebt; sey es Eisen oder Platin; letzteren Falls jedoch betrïcbllich stärker, als ersteren Falls.

Schliefslich noch folgende Bemerkung: Man wird im Vorigen gesehen haben, wie Versuche uber das ungleichzeitige Eintauchen homogener Metalle bei Combination mit anderen Versuchen nützliche Fingerzeige und Bestätigungen für das clektro-chemische Verbältnifs der Flüssigkeiten zu den Metallen liefern können. Für sich allein angestellt, begründen sie gar keinen bestimunten Schlufs, und man kann, wenn man blofs bei ihnen steben bleiben will, die grofsten Anomalien wabrzunebmen glauben, die sich doch bei Combination unit anderen Versuchen sebr woll wechselseitig mit diesem aufklären. In verdünnten Flüssigkeiten ist oft der Ausschlag entgegengesetzt, als in concentrirten, und oft kehrt sich, namentlich in jenen, der erste Ausscblag sogar um. lu nicht seltenen Fällen ist der Ausschlag entgegengesetzt, je nachdem unan kürzere oder längere Zeit zwischen deun Eintauchen der ersten und Zutauchen der zweiten Platte verfliefsen lälst, und diese Resultate sind, unter Beibehaltung derselben Umstände, auch bei Versuchen mit verwechselten Platten, durchaus constant. Dieselbe Flüssigkeit äufsert ferner dabei auf die verschiede. nen Metalle den verschiedensten Einflufs; wie denn z. B. in Kochsalzlösung bei 3 Min. Zwischenzeit das erst ein- 
getauchte Metall negativ ist bei Platin, Silber, Zinn, Blei; positiv bei Kupfer, Eisen, Antimon, bei destillirten Zink endlich aus positiv in negativ umschlagend $u$. s. $f$.

Ich übergehe hier weitere Details; indefs schien es mir nützlicb, diefs hier vorläufig über diesen Gegenstand anzuführen, damit man nicht in der Zeitdifferenz des Eintauchens einen Einflufs suche, der an sich nach einer bestimmten Kichtung wirkt. Alles kounnt darauf an, wie die Veränderungen der Metalle durch die Flüssigkeiten sich im Moment des Eintauchens gestalten, und nach welcher Richtung sie von da fortschreiten. So weit meine bisherigen Versuche reichen, zeigt der Umstand, dafs das erst eingetauchte Metall z. B. positiv wird, stets einen von folgenden beiden Füllen an: entweder die Flüssigkeit ändert das Metall positiv, and diese Aenderung nimmt vom Moment des Eintauchens an zu, oder sie ändert das Metall negativ, und diese Aenderung nimmt vom Moment des Eintauchens an ab. Welcher von beiden Fallen stattinde, läfst sich dann leicht durch Hinzufügung von Versuchen mit dem Condensator und mit Ketten, wo. das Metall mit der betreffenden Fltissigkeit und destillirtem Wasser zugleich combinirt wird, entscheiden. Inzwischen, obschon meine bisherigen Versuche mir keinen Ausnahmsfall von diesen Beziehungen gezeigt haben, sind sie doch noch nicht vervielfätigt genug, um diese Beziehungen schon jetzt als vollkommen und allgemein gesichert festzustellen. 